\section{Progress in the management of patients with uveal melanoma. The 2012 Ashton Lecture}

B Damato

has been enhanced by the formation of multidisciplinary teams in specialised ocular oncology centres.

Eye (2012) 26, 1157-1172; doi:10.1038/eye.2012.126; published online 29 June 2012

Keywords: uveal melanoma; detection; diagnosis; treatment; screening; metastatic disease

\section{Introduction}

Patients with uveal melanoma are faced with many serious threats: visual loss and all the consequences of impaired function; painful ocular morbidity; loss of the eye, with all the social implications of any disfigurement; and early death from cancer. Their care is hampered by the rarity of their disease that is encountered only once or twice in an entire career by their family doctor, once every several years by an optometrist, and only about once a year by their ophthalmologist. They may have to cope with the disappointment of knowing that their tumour was missed when they first sought medical attention, by the frustration that their ophthalmologist was unable to say very much about their condition and its treatment, and by the inconvenience of having to travel repeatedly to a distant hospital for specialised care. They may also suffer because of the lack of adequate psychological support and because of concerns about whether or not they are receiving the best possible care.

Practitioners have to cope with their own shortcomings. Optometrists may experience guilt because they have failed to detect a tumour at the first opportunity, possibly because of compromises they have made in their examination technique. Ophthalmologists
Department of Molecular and Clinical Cancer Medicine, Ocular Oncology Service, Royal Liverpool University Hospital, Prescot St, Liverpool, UK

Correspondence: B Damato, Department of Molecular and Clinical Cancer Medicine, Ocular Oncology Service, Royal Liverpool University Liverpool L7 8XP, UK Tel: + 44 (0)151 7063973; Fax: + 44 (0)151 7065436 . E-mail: Bertil@Damato.co.uk

Received: 23 May 2012 Accepted: 24 May 2012 Published online: 29 June 2012

The 2012 Ashton Lecture was delivered at the Annual Congress of the Royal College of Ophthalmologists in Liverpool on the 16th May 2012. Hospital, Prescot St, 
may feel frustrated at being unable to diagnose tumours and counsel patients adequately. They may also feel apprehensive about having to monitor patients after treatment, especially because of the life-threatening nature of uveal melanoma. Ocular oncologists have to cope with uncertainty when planning patient care, because the required medical evidence on which to base their decisions is lacking. They may also experience stress because of inadequate facilities, compromises that are necessary with patients living far from the oncology centre, and the need to meet national cancer standards in an ophthalmic department. Ocular oncologists also need to cope with failure, with many of their patients dying of their disease despite losing vision and perhaps the eye.

Fortunately, there has been enormous progress in the management of patients with uveal melanoma. The quality of life of these patients is therefore better than might be expected. It would be useful to consider what progress has actually occurred, how this has come about, what obstacles have been overcome, and what needs to be done to improve matters.

Every ocular oncology centre has progressed according to its unique set of opportunities and constraints so that as a result each centre is very different from all others. It would be neither possible nor helpful to attempt to provide a comprehensive, encyclopaedic review of the management of patients with uveal melanoma, which would allow only a bland, superficial overview, lacking insight into the challenges faced by patients and practitioners. This review therefore focuses on the author's personal experience in the management of patients with uveal melanoma, gained over a 28-year period. It would be ideal if all statements and claims could be based on high-level scientific evidence, but the relevant studies have either not been undertaken or are flawed, for reasons which this article will seek to explain.

\section{Uveal melanoma}

Uveal melanomas have an incidence of $\sim 7$ per million per year, being more common in Caucasians than other races. Males and females are affected in equal numbers, but there is a tendency for males to have larger and more posterior tumours. ${ }^{1}$ Presentation nearly always occurs in adulthood, peaking at around the age of 62 years. $^{2}$ Risk factors include fair skin, light-coloured eyes, melanocytoma, congenital ocular melanocytosis, and neurofibromatosis. The role of sunlight is uncertain.

More than $90 \%$ of uveal melanomas involve the choroid, inducing secondary changes in the overlying retinal pigment epithelium (RPE). ${ }^{3}$ These abnormalities consist of proliferation, multilayering, lipfuscin accumulation, drusen, RPE detachments, atrophy, and, rarely, neovascularisation. These changes give amelanotic melanomas their dark appearance, cause autofluorescence, give rise to hyperfluorescence on fluorescein angiography, and induce retinal detachment, with metamorphopsia, blurred vision, photopsia, and visual field loss. Many choroidal melanomas rupture the $\mathrm{RPE}$, prolapsing into the subretinal space, where the herniated tumour becomes strangulated by Bruch's membrane, with the development of venous congestion, oedema, swelling, and a mushroom shape. The tumour can invade and perforate the retina, causing vitreous haemorrhage and, rarely, tumour seeding around the vitreous cavity and anterior chamber. A few choroidal melanomas are diffuse and these are relatively aggressive, and are more likely to extend extraocularly and metastasise.

Approximately 5-8\% of uveal melanomas arise in the ciliary body, extending posteriorly into the choroid, anteriorly into the iris and anterior chamber, and circumferentially. These tumours tend to cause astigmatism and cataract.

Only about $3-5 \%$ of uveal melanomas arise in the iris. They can be nodular or diffuse, the latter tending to seed around the anterior chamber and to extend around the angle causing secondary glaucoma. Diffuse iris melanomas are also associated with increased mortality.

At any stage, the tumour can spread transsclerally, this invasion usually occurring through pre-existing channels for ciliary nerves, veins, arteries, and aqueous drainage channels. ${ }^{4}$

Metastatic tumour dissemination occurs haematogenously. Lymphatic spread to regional lymph nodes is extremely rare, occurring only if there is extraocular tumour growth into the episclera. In almost all patients, metastatic disease first becomes apparent in the liver, less common sites being the lung, bone, and skin. The risk of metastatic disease correlates with many clinical, histological, and genetic factors, which are discussed later in this article. Almost all patients die within a year of the onset of symptomatic metastatic disease, the survival time correlating with factors such as size and number of hepatic metastases, liver function test results, Karnowski index, and time interval between ocular treatment and onset of metastatic disease. ${ }^{5}$

\section{Aspects of patient management}

Management involves tumour detection; diagnosis; patient counselling and care planning; treatment of the ocular tumour; detection and treatment of local tumour recurrence and other ocular complications; prognostication; psychological support; screening for metastatic disease, if appropriate; and treatment of metastases. This is best delivered by a multidisciplinary team comprising ocular oncologists, vitreoretinal 
surgeons, anaesthetists, specialist nurses, photographers, secretaries, pathologists, oncologists, radiation physicists, psychologists, database managers, and others. In the United Kingdom, supra-regional services specialising in adult ocular oncology exist in the Glasgow, Liverpool, London, and Sheffield thanks to the government funding provided in the National Health Service through the National Specialist Commissioning Group in England and the Scottish Home and Health Department. The oncology centres are funded according to the number of patients managed but are required to comply with the national cancer standards, to provide activity and outcomes data, and to hold a joint meeting annually. The Liverpool Ocular Oncology Centre (LOOC) was established by the author in 1993 and receives $>700$ patients each year, with $\sim 250$ of these having an ocular melanoma.

\section{Detection}

As with other cancers, early detection and treatment of uveal melanoma should enhance any opportunities for conserving the eye and vision and for prolonging life. Smaller tumours require less extensive radiation to the eye, for example, and are less likely to extend close to the optic disc and fovea. Damato and Damato ${ }^{2}$ have therefore audited the detection and referral of 2384 patients, who were diagnosed with uveal melanoma at the LOOC between 1996 and 2011. The referral process was initiated by an optometrist in $68 \%$ of patients, a general practitioner in $18 \%$, and an ophthalmologist in $14 \%$. On referral, $30 \%$ of patients were asymptomatic and these patients were more likely to retain the eye $(87 \%$ vs $\left.58 \% ; \chi^{2}, P<0.001\right)$. Conversely, $23 \%$ of symptomatic patients said that their tumour had initially been missed; these patients experienced longer delays in treatment (median: 92 vs 40 days; Mann-Whitney, $P<0.001$ ). When first seen at our centre, patients reporting that their tumour was initially missed were more likely to have: reduced vision $\left(\chi^{2}, P<0.001\right)$; greater basal tumour diameter (Mann-Whitney, $P<0.001$ ); optic disc involvement by the tumour $\left(\chi^{2}, P=0.033\right)$; and extraocular spread $\left(\chi^{2}, P=0.015\right)$. For these reasons, they were also more likely to require primary enucleation (45\% if initially missed $v$ s $30 \%$ if promptly detected; $\left.\chi^{2}, P<0.001\right)$.

This audit indicates that there is scope for improvement in the detection of uveal melanoma, particularly by optometrists in the community. Patients are not always receiving the 'full eye examination' they expect, and which they possibly feel they are paying for. More widespread use of fundus photography should improve this situation, especially if wide-angle cameras are used. Until mydriasis is performed routinely, the
Table 1 Symptoms and external signs indicating the presence of an intraocular melanoma.

\begin{tabular}{ll}
\hline Mneomonic & Description \\
\hline $\mathrm{M}$ & Melanoma visible externally, in the iris or episclera \\
$\mathrm{E}$ & Eccentric visual phenomena (eg, photopsia) \\
$\mathrm{L}$ & Lens abnormalities (cataract, astigmatism) \\
$\mathrm{A}$ & Afferent papillary defect \\
$\mathrm{N}$ & No optical correction with spectacles \\
$\mathrm{O}$ & Ocular hypertension \\
$\mathrm{M}$ & Melanocytosis, predisposing to melanoma \\
$\mathrm{A}$ & Asymmetrical, episcleral 'sentinel' vessels \\
\hline
\end{tabular}

author has prepared a mnemonic listing the clinical features associated with an intraocular melanoma (Table 1).

\section{Referral}

The detection and referral audit showed that the median delay from the first assessment by an ophthalmologist to the time when the referral communication was received at the LOOC was 7 days; however, in 252 (11\%) patients this delay was $>6$ months. $^{2}$ In this subset, the basal tumour diameter exceeded $12 \mathrm{~mm}$ in $13 \%$ of patients when they were eventually assessed at the oncology centre, with $26 \%$ of tumours exceeding $4 \mathrm{~mm}$ in thickness, $19 \%$ involving the optic disc, $7 \%$ extending extraocularly, and $28 \%$ showing chromosome 3 loss. Primary enucleation was required in $28 \%$ of these patients.

The most likely explanation for delayed referral of patients by the ophthalmologist to the ocular oncologist is misdiagnosis. It is difficult for ophthalmologists to remember the clinical features of rare tumours, which they might encounter only once every few years. The author has created an online atlas, which organises tumours and pseudo-tumours according to their colour and location in the eye (http://www.oculonco.com). Assessment of tumour colour is best made by slit-lamp examination or ophthalmoscopy, because not all cameras reproduce colour accurately. When distinguishing choroidal naevus from melanoma, it is helpful to perform autofluorescence photography and optical coherence tomography so as to detect lipofuscin 'orange pigment' and serous retinal detachment, which suggest malignancy. It is also useful to measure tumour thickness by ultrasonography, placing the calliper at the internal scleral surface and taking account of any overlying retinal detachment, also ensuring that the probe is held at right angles to the scleral and tumour surfaces. A baseline colour photograph is essential, ensuring that all the tumour margins are included in the image(s). Patients who are kept under surveillance at the local hospital must be informed about the possibility of 
malignancy and given advice as to what to do should their follow-up schedule be disrupted for any reason. ${ }^{6}$ When patients are referred with suspected tumour growth, after a period of surveillance, it is useful for the referring ophthalmologist to send the ocular oncologist a copy of the baseline colour photograph of the tumour. Patients should be advised by the referring ophthalmologist what to do if no communication from the oncology centre is received within 2 weeks. Optometrists undertaking surveillance of any patient with a possible melanoma should provide the same standards of care as those expected from an ophthalmologist (eg, photography, counselling, letter to the family doctor after each visit, information sheets, precautions against loss to follow-up, so on).

\section{Initial management at ocular oncology centre}

The objectives of this stage of the care pathway are to see all patients within 2 weeks of receipt of the referral and to treat as many patients as possible within a day of their first assessment at the LOOC, providing a one-stop service, but ensuring that all patients receive the information, counselling, and emotional support that they require. Although some might question the ethics of treating patients within a day of their first assessment at an oncology centre, an audit at the LOOC has shown that the large majority of patients want immediate treatment, only a few requiring more than a day to reflect on their condition and select their preferred management. ${ }^{7}$

\section{Referral procedure}

A useful development has been to send all new patients a 70-page guidebook, covering aspects such as: getting to the oncology centre; how care is organised; ocular tumours and treatments; and sources of information and support. This prepares them for their first meeting with the ocular oncologist, making it easier for them to understand what they are told. To prevent the patients with non-malignant conditions from being alarmed unduly, repeated reassurances are given that most patients referred to an ocular oncology centre do not have a cancerous tumour. Patients also receive a consent form and information sheet so that data and images can be collected for research, teaching, and audit without delay.

\section{Investigation}

Visual acuity is measured with the LogMar chart, recording the visual acuity rating, with a score of 100 corresponding to perfect vision and recording visual acuity $<6 / 60$ as counting fingers. These modifications enable all patients to have LogMar acuity measured in a busy, routine clinic. Patients are examined by an ophthalmic surgical trainee, who organises appropriate imaging, consisting of wide-angle fundus photography and/or anterior segment photography, with optical coherence tomography and autofluorescence photography in selected cases. The history and all clinical findings are recorded on special proformas in the casenotes, minimising free text, to facilitate subsequent computerisation by a full-time data manager. On meeting the patient, the ocular oncologist reviews the notes and images, examines the patient, performs ultrasonography, counsels the patient, and dictates a letter to the referring ophthalmologist, with copies to the family doctor, optometrist, and the patient. Fluorescein and/or indocyanine green angiography are not routinely performed. If the clinical diagnosis is uncertain, a tumour biopsy is performed the next day or, if more appropriate, arrangements are made for long-term surveillance at the LOOC or the referring hospital, or alternating between the two. Screening for metastatic disease is not performed unless the basal tumour diameter is $17 \mathrm{~mm}$ or more, in which case liver ultrasonography is performed, before the ocular treatment is started or as soon as possible afterwards, ensuring that this investigation does not delay ocular surgery. It is explained to the patient that a large uveal melanoma needs to be treated in any case, to prevent a painful crisis, whether or not liver metastases are present. The author's view, which is not widely held, is that screening of all patients would detect many harmless abnormalities, which would complicate care. In a cohort of 90 patients screened with computerised tomography, 50 patients were found to have hepatic abnormalities, which were suspicious of metastases in 6 but that were confirmed as secondaries in only 3 patients. ${ }^{8}$

\section{Patient counselling}

The ocular oncologist orally informs patients about ocular anatomy; the nature of their condition; what might happen without treatment; the therapeutic options; the advantages and disadvantages of each modality; the likely outcomes; and possible side effects and complications. This interactive discussion is facilitated by fundus photograph on a large screen (consisting of a wide-angle, 3D image if the tumour is choroidal) and a plastic model of the eye. An audio recording of the conversation is subsequently given or mailed to the patient on a cassette tape or a CD-ROM, according to the patient's preference. ${ }^{9}$ After meeting the ocular oncologist, the patient moves to an adjacent room where a specialist ocular oncology nurse checks that the patient has understood all that was said, also providing explanations about the logistics of treatment, aftercare, and other matters. Information sheets relating to the 
selected management are provided to the patient. The specialist nurse also organises all pre-operative investigations. Patients are visited by a health psychologist, who screens them for any problems, if necessary organising appropriate support once they return home. On discharge from hospital, patients are given an information pack and an anonymous satisfaction questionnaire. Soon after returning home, they are phoned by the specialist oncology nurse who provides support and reminds them about her telephone helpline, which they can call at any time.

\section{Ocular treatment}

\section{Scope}

It is still not known whether ocular treatment influences survival and, if so, in whom. ${ }^{10}$ As long ago as 1868 , Knapp ${ }^{11}$ wrote that the assertion that early treatment will prevent metastasis that has 'long since, and until this day, been attacked and defended from many sides'. Manschot ${ }^{12}$ reported histopathological evidence of tumour viability after radiotherapy and argued that all patients with uveal melanoma should undergo immediate and urgent enucleation. Zimmerman ${ }^{13}$ hypothesised that enucleation accelerated metastatic death. To settle the Zimmerman-Manschot controversy, large multicentre, randomised trials were conducted by the Collaborative Ocular Melanoma Study (COMS) in the United States. One of these studies aimed to establish whether plaque radiotherapy of uveal melanoma was as 'safe' as enucleation, and the other investigated whether pre-enucleation radiotherapy improved survival in comparison with enucleation alone. ${ }^{14,15}$ It is generally held that no survival differences were found in these two COMS trials. However, both studies included patients who definitely had pre-existing micrometastases at the time of ocular treatment, because they had died of metastasis within the first 3 post-operative years. If these patients had been excluded, on the grounds that ocular treatment could not possibly have prevented spread that already occurred, then neither study would have had enough patients to attain sufficient statistical power. In other words, the COMS trials were inconclusive. ${ }^{16}$ Even if no significant differences in survival had been demonstrated statistically, these studies would not have revealed whether the rival ocular treatments were equally effective or equally ineffective at preventing metastatic death.

On the basis of tumour doubling times, Eskelin et al ${ }^{17}$ have hypothesised that metastatic spread commences years before the primary ocular tumour is detected and treated. As metastatic death correlates strongly with chromosome 3 loss, it would seem that tumour dissemination from the eye commences at the same time or very soon after chromosome 3 loss occurs. ${ }^{18}$ Höglund et $a l^{19}$ have proposed that in melanomas karyotypic abnormalities follow defined pathways, starting either with either chromosome 3 loss or chromosome 6p gain. Tschentscher et $a l^{20}$ have speculated that lethal and nonlethal variants of uveal melanoma are distinct from their inception. These studies suggest that chromosome 3 loss and metastatic spread occurs very early in the development of uveal melanomas, so that by the time the patient presents, it is too late for any ocular treatment to prolong life.

The Liverpool Ocular Oncology Research Group ${ }^{21}$ studied abnormalities in chromosomes 1, 3, 6, and 8 in 452 uveal melanomas and found that the accumulation of chromosomal abnormalities occurred in a more variable and random sequence than suggested by Höglund et al. Furthermore, although there was a trend towards more numerous chromosomal abnormalities in larger tumours, there was much variation between patients. In other words, some smaller tumours showed more numerous chromosomal abnormalities than large tumours. These findings indicate that lethal chromosomal abnormalities and metastatic spread occur early in some patients and late in others. Callejo et $a l^{22}$ reported a patient whose choroidal melanoma suddenly grew markedly after several years of indolent activity, developing a collarstud shape, and disseminating tumour cells widely throughout the eye. Histological and genetic analysis of the enucleated eye showed low-grade, disomy 3 melanoma in the older, basal region of the tumour and high-grade, monosomy 3 melanoma in the more recent, apical part of the tumour. After this paper was published, the patient died of metastatic disease. This case suggests that transformation from low-grade to high-grade melanoma and metastatic spread can occur late, and are therefore preventable in some patients. The main implication of these findings is that delayed treatment of uveal melanoma and recurrent tumour after inadequate treatment may therefore shorten life in some patients. Furthermore, whereas there may be a tendency to treat large uveal melanomas more urgently and aggressively, it is perhaps the small tumours that should be treated most urgently and aggressively because it is with early disease that any opportunities for preventing metastatic spread are greatest.

In theory, there is scope for a randomised trial of treatment vs non-treatment of asymptomatic uveal melanomas; in practice, such a study would be complicated by ethical concerns, by the reluctance of many patients to leave a malignant tumour untreated, and by a high dropout rate caused by the onset of symptoms. 


\section{Phototherapy}

Photocoagulation dates back to 1949, when Meyer Schwickerath and Vogel $^{23}$ used lenses to focus the sun's rays onto a choroidal melanoma. This was followed by xenon arc and Argon laser photocoagulation, delivering very brief bursts of high-intensity light to the tumour. Such photocoagulation had a high-rate of complications, which included retinal traction and detachment, vascular occlusion, choroidal neovascularisation, and local tumour recurrence. Foulds and Damato ${ }^{24}$ developed low-intensity, long-duration laser treatment, using krypton laser, which had greater penetrance. This concept was developed further by Oosterhuis, ${ }^{25}$ when infrared lasers became available, calling his technique transpupillary thermotherapy (TTT). The principle of low-energy phototherapy is to heat the tumour by a few degrees for about $1 \mathrm{~min}$ so as to disrupt the metabolic processes of the tumour cells. TTT is more effective and safer than photocoagulation but is associated with a significant rate of local tumour recurrence and is therefore used mostly as an adjunct to radiotherapy. In 1985, Damato and Foulds treated two patients with photodynamic therapy with an effective tumour response but with excessive photosensitivity (unpublished data). The development of Verteporfin in the 1990s, encouraged Heimann and Damato (and others elsewhere) to treat more patients with photodynamic therapy. ${ }^{26}$ This treatment is still under investigation.

\section{Radiotherapy}

Radiotherapy of uveal melanomas requires high doses of radiation to be delivered to the tumour while avoiding collateral damage to surrounding, healthy tissues.

Brachytherapy Brachytherapy for uveal melanoma was first performed in 1930 by Moore, ${ }^{27}$ who implanted a radon seed into the tumour. Subsequently, brachytherapy was administered with a variety of saucer-shaped plaques. Initially, cobalt plaques were used, but these were superseded by applicators containing iodine or ruthenium. Iodine plaques emit gamma radiation, which has a long range making it possible to treat large tumours, albeit causing significant morbidity in such eyes. Ruthenium plaques emit beta radiation, which has a shorter range, causing less collateral damage but reliably treating only smaller tumours. The conventional practice is to position the plaque so that it overlaps the entire tumour margins by $2 \mathrm{~mm}$. With posterior tumours, this wide safety margin increases the likelihood of radiation-induced optic neuropathy and maculopathy. Some may therefore be tempted to reduce the dose of radiation as much as possible, albeit with a greater risk of
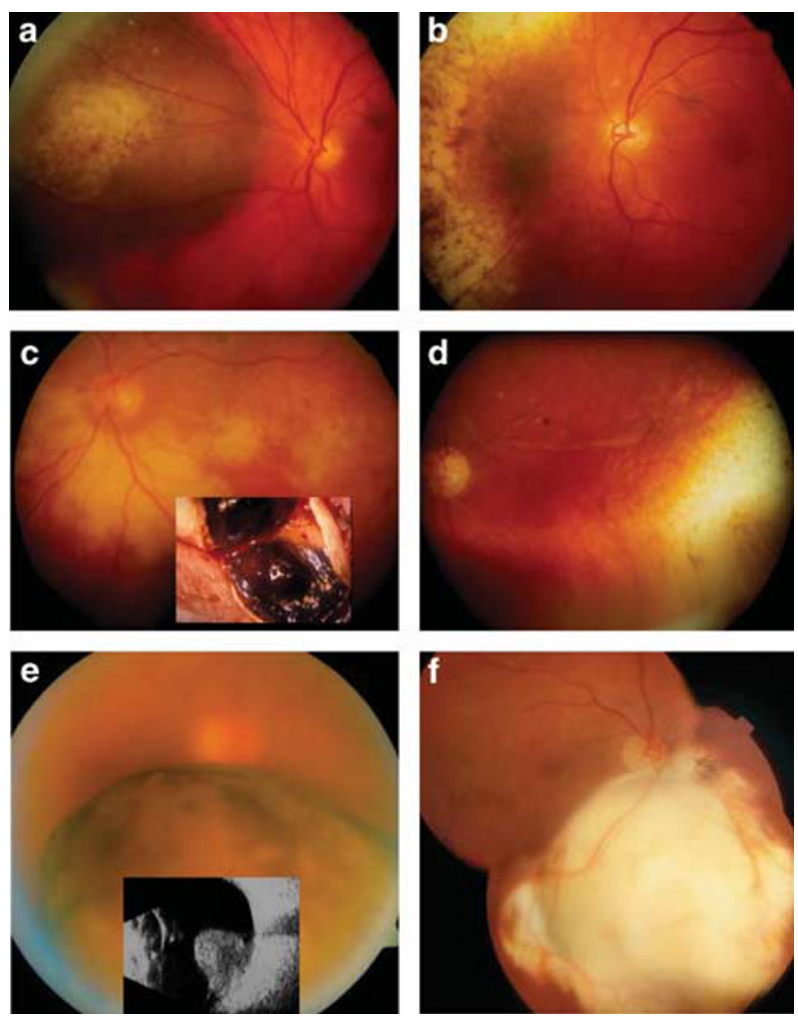

Figure 1 (a) Choroidal melanoma in the left eye of a 40-yearold woman treated with an eccentrically positioned, ruthenium plaque in 1986; (b) Fundus appearance 3 years later. Ten years post-operatively, the visual acuity was $6 / 6$ and 26 years after treatment the patient was still alive. (c) Exudative retinopathy after ruthenium plaque radiotherapy of a ciliary body melanoma in the left eye of a 51-year-old man with diabetes. The 'toxic tumour' was excised in 1989. (d) Fundus appearances 3 years later, showing resolution of the exudation. (e) Inferior, juxtapapillary, choroidal melanoma with a basal diameter of $10.4 \mathrm{~mm}$ and a thickness of $9.6 \mathrm{~mm}$, in the right eye of a 65-year-old man. The patient was treated by endoresection in 1999. (f) Postoperative fundus appearance. Thirteen years after surgery, the visual acuity with the operated eye was $6 / 5$. All procedures performed by the author.

local treatment failure. The author has developed instruments and techniques for eccentric plaque placement, aligning the posterior edge of the plaque with the posterior tumour margin (Figures $1 a$ and $b$ ). As the plaque is located further from the optic disc and fovea, higher doses of radiation can be delivered without damaging these structures. Audits performed by Russo et al and Damato et al show good rates of visual conservation, with low recurrence rates. ${ }^{28-30}$

Proton beam radiotherapy The Clatterbridge Centre for Oncology is one of only a dozen or so centres around the world providing proton beam radiotherapy for ocular tumours, and the only facility of its kind in the 
United Kingdom, which is why the author moved from Glasgow to Liverpool in 1993. Proton beams are highly collimated and their destructive ionising radiation peaks at the depth where the charged particles stop travelling (ie, the 'Bragg peak'). It is therefore possible to target the radiotherapy very precisely at the tumour, with good sparing of surrounding tissues.

As with other studies, the Liverpool audit showed high rates of local tumour control; however, there was appreciable loss of vision even when the optic nerve and fovea received minimal or no radiation. ${ }^{31}$ This visual loss correlated with tumour size, as did secondary enucleation, which was required because of painful neovascular glaucoma. Daftari et $a l^{32}$ suggested that this morbidity was caused by radiation of healthy ciliary body and iris. The author discovered, however, that exudative retinal detachment, rubeosis, and neovascular glaucoma resolved if the irradiated tumour was excised. He coined the term 'toxic tumour syndrome' to describe complications caused by the persistence of an ischaemic and exudative tumour in the eye after radiotherapy (Figures 1c and d). Depending on the size of this toxic mass and the severity of the complications, patients can be treated with intraocular steroids and/or antiangiogenic agents, TTT, photodynamic therapy, endoresection, or transscleral local resection. There has also been progress in the avoidance of 'direct' radiationinduced complications, such as the development of the notched beam for juxtapapillary tumours. Another advance has been to treat superior tumours though the closed upper eyelid to avoid radiation of the eyelid margin, which causes keratinisation of the superior tarsal conjunctiva, painful keratopathy, and even corneal perforation.

Another development has been proton beam radiotherapy of iris melanomas, which avoids the cosmetic and functional problems of an extensive surgical iris coloboma and which provides better radiation dosimetry than brachytherapy. An audit by the author has shown that most patients retain their pre-treatment visual acuity although many have required treatment for cataract and glaucoma. ${ }^{33}$ Local tumour recurrence has been rare (ie, $<5 \%$ ), occurring mostly with diffuse iris melanomas whose extent was underestimated by initial slit-lamp examination. Such tumours are now treated by proton beam radiotherapy to the entire anterior segment. ${ }^{34}$ So far, $\sim 16$ patients have received such extensive radiotherapy in Liverpool, and the results are currently being audited. Some patients have developed discomfort from mild or moderate limbal stem cell failure but symptoms have been tolerable with medical therapy. No patients have required limbal stem cell transplantation from the fellow eye. All these eyes have been conserved, which is encouraging as all would have needed enucleation in the first instance if not treated by radiotherapy.

Surgical resection There has been considerable progress in the surgical resection of uveal melanomas since the author started practising adult ocular oncology in 1984.

Iridectomy results have improved with the development of artificial iris implants, pupilloplasty, and painted contact lenses.

Iridocyclectomy techniques currently performed by the author have changed in the past decade. Previously, the pupil was dilated and the dissection was performed anteroposteriorly, commencing with a broad iridectomy. Today, the author constricts the pupil and excises the tumour in a postero-anterior or circumferential direction, conserving the iris sphincter and most of the iris in the affected sector, leaving only a small peripheral iridectomy. This improvement has resulted in a swing away from radiotherapy and back to surgical excision, which provides not only a cure but also tissue for diagnosis and prognostication. ${ }^{35}$

Choroidectomy can be performed transsclerally (ie, 'exoresection') or transretinally (ie, 'endoresection') and can be primary or secondary, the latter being undertaken as a treatment for local tumour recurrence or the toxic tumour syndrome after radiotherapy.

Exoresection is performed by excising the tumour en bloc together with the adjacent deep scleral lamella, if possible without damaging the retina and using the superficial scleral lamella to close the eye. Haemorrhage is reduced by lowering the blood pressure to $\sim 50 \mathrm{~mm} \mathrm{Hg}$. Previously, exoresection was performed only as a salvage procedure if radiotherapy had failed, but in the 1970s Foulds ${ }^{36}$ started performing this operation as a primary procedure and irrespective of the vision in the fellow eye. Early results have been audited. ${ }^{37-40}$ Improvements in exoresection include better scleral flap design (ie, polyhedral shape facilitating closure); safer perforation of deep sclera, avoiding uveal damage (by shaving instead of incising); safer uveal perforation, avoiding retinal damage (by ripping the choroid with notched microforceps instead of incising); avoidance of retinal prolapse through the scleral window (with ocular decompression by core vitrectomy); safer separation of the retina from adherent tumour (by using a Bard Parker scalpel instead of blunt dissection); better haemostasis (by closure of the short posterior ciliary arteries near the optic nerve); safer closure of scleral flap (with non-absorbable nylon sutures); avoidance of local tumour recurrence (by adjunctive brachytherapy, using a 25 -mm ruthenium plaque); prevention of retinal detachment if a retinal tear occurs (by immediate 
vitreoretinal surgery, using silicone oil); prevention of hypotony after cyclectomy (by delaying adjunctive brachytherapy for 1 month); and prevention of retinal dialysis and detachment after cyclo-choroidectomy (by commencing tumour resection posterior to ora, making it possible to conserve the ciliary epithelium over the pars plana). These technical refinements have considerably improved the results of transscleral choroidectomy so that the procedure is now completed within $2 \mathrm{~h}$, with rare intra-operative complications. A recent audit of 112 such procedures performed between 2000 and 2010 showed that $88 \%$ of eyes were conserved with $58 \%$ having visual acuity of $6 / 60$ or better and $30 \%$ retaining acuity of $6 / 12$ or better (unpublished data). Local recurrence occurred in 11 patients (only 3 of whom had received adjunctive brachytherapy). Retinal detachment developed in 9 eyes. All these tumours had a median basal diameter of $15.3 \mathrm{~mm}$ and a median thickness of $8.5 \mathrm{~mm}$ so that most would have been treated by primary enucleation if local resection had not been possible.

Endoresection of choroidal melanoma involves removal of the tumour with a vitreous cutter, either through a retinotomy overlying the tumour or after raising a retinal flap. Fluid-air exchange is performed to flatten the retina and to allow laser retinopexy. Phototherapy is also administered to destroy any tumour remnants in the sclera. To prevent post-operative haemorrhage and retinal detachment, the eye is filled with silicone, which is removed after 12 weeks. Adjunctive plaque or proton beam radiotherapy is administered if histology indicates a high-grade melanoma. Preliminary results after the first 52 endoresections were reported by the author and associates in 1998 (Figures 1e and f). ${ }^{41}$ A more up-to-date evaluation is in progress now that $>60$ further procedures have been undertaken. The main complications have been those of vitrectomy. Local tumour recurrences have been rare with extraocular extension, widespread intraocular seeding, and subconjunctival seeding each occurring in only one patient. ${ }^{42,43}$ Some authors advocate neoadjuvant radiotherapy; ${ }^{44,45}$ however, the Liverpool results suggest that only a few patients would benefit from such additional treatment, most experiencing unnecessary radiation-induced morbidity.

The author's enucleation technique has changed considerably in recent years. Improvements include: abandoning Castroviejo implants, which commonly extruded; administering a long-acting local anaesthetic with adrenaline, after general anaesthesia has been induced, to reduce intra-operative haemorrhage and post-operative pain; applying a pressure bandage for 2 days, to prevent post-operative peri-ocular haematoma; and counselling patients about phantom eye symptoms, which are common and diverse. The Castroviejo implants were initially replaced with $22 \mathrm{~mm}$ coral implants wrapped in donor sclera, but a randomised, prospective trial is now in progress comparing FCI alumina implants with acrylic implants, the latter containing a nylon suture to which the muscles are attached. To date, $\sim 400$ patients have been randomised, but interim studies indicate little difference between the two implants, despite the great difference in cost (unpublished data). For several years, enucleations in Liverpool have been performed using a kit entirely consisting of disposable instruments, which we commissioned in case the use of re-usable instruments is ever banned because of concerns about prion disease. Damato and Lecuona ${ }^{46}$ devised a prognostic score, estimating the likelihood of primary and secondary enucleation according to basal tumour diameter, tumour thickness, optic disc involvement, and coronal tumour location. Primary enucleation continues to be necessary in over a third of patients, because of late presentation, detection or referral.

Over the years, there has been greater use of multimodal therapy, such as local resection with adjunctive radiotherapy and radiotherapy with adjunctive phototherapy.

\section{Follow-up}

After ocular treatment, arrangements are made for patients to be reviewed at their local hospital to ensure that there are no early post-operative complications. Follow-up is then alternated between the LOOC and the referring hospital until the risk of local tumour recurrence is estimated to be $<1 \%$, when the patient is discharged to the referring unit. Ophthalmologists are encouraged to refer patients back to the oncology centre at any time if any concerns ever arise. A Nurse Oncology Clinic has been established at the LOOC for long-term surveillance of low-risk patients who do not need to see an ophthalmologist. ${ }^{47}$ British patients with a primary ocular malignancy are immediately flagged according to their National Health Service (NHS) number at the NHS Cancer Registry, which automatically informs the LOOC of the date and certified cause of all deaths.

\section{Prognostication}

Survival prognostication enables reassurance of patients with a good survival probability; targeting of special care such as counselling, screening and systemic adjuvant therapy at high-risk patients; planning of clinical trials; and laboratory research into survival predictors and tumour biology. ${ }^{48}$ 


\section{Clinical predictors}

The main clinical features correlating with increased mortality are: largest basal tumour diameter; tumour thickness; ciliary body involvement; and extraocular spread. Largest basal tumour diameter is the indicator that is most widely used, but there is much variation in the way this is measured, some relying on ultrasonography and others preferring ophthalmoscopy. ${ }^{49}$ The basal diameter can be underestimated if the tumour has diffuse margins or if there is annular growth around the ciliary body. Some authors consider tumour thickness to be a good predictor of survival; 50 however, thickness reflects not only neoplastic growth but also tissue swelling if the tumour has prolapsed through Bruch's membrane (see above). It is not known why large tumours are associated with increased mortality, because it is not known whether metastatic spread commences before or after tumour growth. It is possible that large tumour size reflects rapid growth, because of detection bias. For example, if a small choroidal melanoma is missed by an optometrist at a 2-yearly assessment, then if it is of low-grade malignancy it is likely to be slow-growing and to remain quite small 2 years later when the patient presents for another checkup; however, if it is of high-grade malignancy, it is likely to grow quickly and to become large and symptomatic before the next assessment. Detection bias may also explain why ciliary body involvement correlates with increased metastatic mortality. It is possible that high-grade, anterior choroidal melanomas grow to involve the ciliary body and become symptomatic whereas many low-grade tumours involving the ciliary body remain small without ever being detected during the patient's lifetime. In keeping with this hypothesis is the finding that ciliary body involvement loses statistical significance when multivariate analysis includes histological and genetic risk factors (unpublished data). The same loss of significance occurs with extraocular extension, suggesting that this feature correlates with increased mortality only because it is associated with high-grade malignancy and lethal genetic abnormalities. ${ }^{4}$ It is these features that cause metastasis and not the fact that the tumour has breached the sclera.

The American Joint Committee on Cancer has recently revised the TNM (tumour, node, metastasis) staging system for uveal melanoma with data on 7867 patients from Berlin, Liverpool, Lyon, Paris, and Helsinki. ${ }^{51}$ The ciliary body and choroidal tumours are categorised according to their basal diameter, thickness, ciliary body involvement, and extraocular extension. Such anatomical staging provides only a crude indication of the survival probability, which is relevant to large groups of patients and not individual patients. As mentioned below, mathematical methods have been developed to combine the TNM stage with pathological and genetic prognostic factors.

\section{Pathological predictors}

The main histopathological predictors of metastatic death include epithelioid melanoma cytomorphology; the presence of extravascular matrix patterns such as closed loops; and high mitotic count per 40 high-power fields. ${ }^{48}$ Many other features correlate with increased mortality and these include increased lymphocytic infiltrate; increased microvascular density; loss of immunoreactivity for HSP-27 and loss of BRCAassociated protein 1 staining. ${ }^{52,53}$ Whether these are of prognostic significance when included in a multivariate model is still uncertain. There are several limitations in the histological grading of malignancy. First, it can be difficult to distinguish spindle melanoma cells from epithelioid cells. Second, there is no consensus as to how many epithelioid cells should be present for the tumour to be classified as mixed or epithelioid. Third, mitoses can be difficult to detect and to distinguish from apoptotic nuclei, and aids such as Ki-67, Her-10 are not widely used. ${ }^{54}$ Fourth, extravascular matrix patterns can be difficult to recognise unless the periodic acid Schiff stain is used, without counterstain. Despite these limitations, most histopathological indicators of histological grade of malignancy show good correlation with metastatic mortality. Ocular pathologists in Liverpool have for several years used synoptic reports, in addition to free text, to avoid ambiguity.

\section{Genetic predictors}

In 1980s, it was discovered that uveal melanomas tend to develop several non-random chromosomal abnormalities and that these correlate with survival. The most important are chromosome 3 loss 'monosomy 3'; chromosome 1p loss; chromosome $6 \mathrm{p}$ gain, and chromosome $8 \mathrm{q}$ gain. ${ }^{48}$ Chromosome 3 loss can be partial, with one or more microdeletions, or total, with loss of the entire chromosome. Rarely, the chromosome 3 loss is a functional loss, there being two chromosomes, but both from the same parent ' isodisomy 3'. Gains in chromosome $6 \mathrm{p}$ develop when the chromatids split transversely during cell division, instead of longitudinally, so that both short arms are pulled by the mitotic spindle into the same cell (ie, isochromosome $6 \mathrm{p}$ ). Isochromosome $8 \mathrm{q}$ occurs in a similar manner. On occasion, there can be trisomy 8 . These abnormalities develop because of genomic instability during cell division, possibly because the spindle is abnormal, 
or because a mitotic checkpoint is dysfunctional. Metastatic death develops almost exclusively with uveal melanomas showing chromosome 3 loss, such tumours showing a high mortality. ${ }^{18}$ In the few patients who die without apparent chromosome 3 loss, it is not known whether this is because the laboratory tests fail to detect the lethal abnormality or whether metastatic spread can occasionally happen without this chromosomal defect.

Mortality is much worse when chromosome 3 loss and 8 gain occur together, as they usually do. ${ }^{21}$ In the few patients who die without apparent chromosome 8 gain it is possible that lethal defects are missed. Chromosome $6 \mathrm{p}$ gain is associated with a relatively good prognosis, apparently delaying or preventing chromosome 3 loss and, in the presence of concurrent chromosome 3 loss, delaying metatastatic death. ${ }^{21}$

Soon after Prescher et al published their landmark paper in 1996, correlating chromosome 3 loss with mortality, the LOOC employed a full-time basic scientist, who confirmed the prognostic value of cytogenetics. In 1999, the LOOC started offering genetic testing to patients as a routine, clinical prognostic service. To the author's knowledge, this had never been done before. It was necessary to establish new guidelines and protocols regarding patient consent, informing patients of the results, and on psychological support and counselling. For the first few years, fluorescence in situ hybridisation (FISH) was used, but an audit showed that this was not sensitive enough to detect small chromosomal abnormalities. ${ }^{55}$ Fortunately, a technique known as multiplex ligation-dependent probe amplification (MLPA) had become available but although a commercial kit had been developed specifically for uveal melanoma it had not been adequately validated. This validation was done in Liverpool where MLPA quickly replaced FISH, proving more sensitive and informative, less expensive, requiring smaller tissue samples, and working with formalin-fixed, paraffin-embedded specimens. ${ }^{56}$ Briefly, MLPA simultaneously identifies gains and losses of 31 genetic loci on chromosomes 1p, 3, 6p, and 8q. The Liverpool Ocular Oncology Research Group, which had recently been established by Coupland and the author, also determined the significance of intra-tumoural genetic heterogeneity and developed computerised methods for interpreting control results. ${ }^{56-58}$ Audits confirmed the clinical value of MLPA, showing low rates of metastasis in the absence of chromosome 3 loss. $^{21}$ These results encouraged us to extend our prognostic service so that we started performing biopsy on tumours being treated by radiotherapy or phototherapy. This required the deployment of microsatellite analysis (MSA) for tumour specimens that were too small for MLPA. ${ }^{59}$ The results of MSA are encouraging but are awaiting further audit, which will be undertaken once there are sufficient data for meaningful analysis.

Other centres have as adopted genetic prognostic typing of uveal melanomas, using a variety of techniques. In the United States, Harbour's team developed a test based on gene expression profiling (GEP), categorising uveal melanomas as class 1 and class 2, respectively. ${ }^{60,61}$ Despite initial reports that class 1 melanomas are non-lethal, metastatic deaths occurred in some patients, so that a class $1 \mathrm{~b}$ category was belatedly defined. GEP is many times more expensive than MLPA, but apparently requires smaller samples. Whether it is otherwise superior to MLPA has yet to be determined by independent studies.

\section{Multivariate analysis}

The author and colleagues have developed an online program that estimates prognosis according to clinical, histological, and genetic risk factors also taking account of the patient's age and sex (http://www. ocularmelanomaonline.com). ${ }^{62}$ This was developed with data on 3653 British patients treated in Liverpool. Validation studies showed good correlation between predicted and actual outcomes in the different risk groups. These results indicate that the Liverpool Uveal Melanoma Prognosticator Online (LUMPO) gives prognostications that are accurate enough to be relevant to individual patients. The program produces two survival curves: an all-cause mortality curve for patients with uveal melanoma and a survival curve for the general British population, matched for age and sex. By subtracting one curve from the other, it is possible to estimate both the metastatic mortality and the mortality from competing risks (ie, unrelated disease). This 'Hakulinen method' is superior to the Kaplan-Meier analysis in that it does not depend on certified cause of death, which is known to be mistaken in many patients (eg, cardiac arrests causes by metastases to heart). ${ }^{63}$ Furthermore, it adjusts for bias caused by competing risks thereby giving a true indication of the chances of dying of metastatic disease (Figure 2). For example, the Kaplan-Meier analysis suggests that metastatic mortality increases with age, but this impression is false and occurs because all unrelated deaths are censored, leaving only metastatic deaths, the importance of which is therefore exaggerated. Conversely, the Hakulinen method shows that in old age the chances of metastatic death diminish as more patients die of unrelated causes before metastatic disease develops.

Personalised prognostication is controversial because it is widely felt that there is no point in predicting a fatal outcome that is essentially unpreventable, thereby causing 'unnecessary' stress to patients. The author 


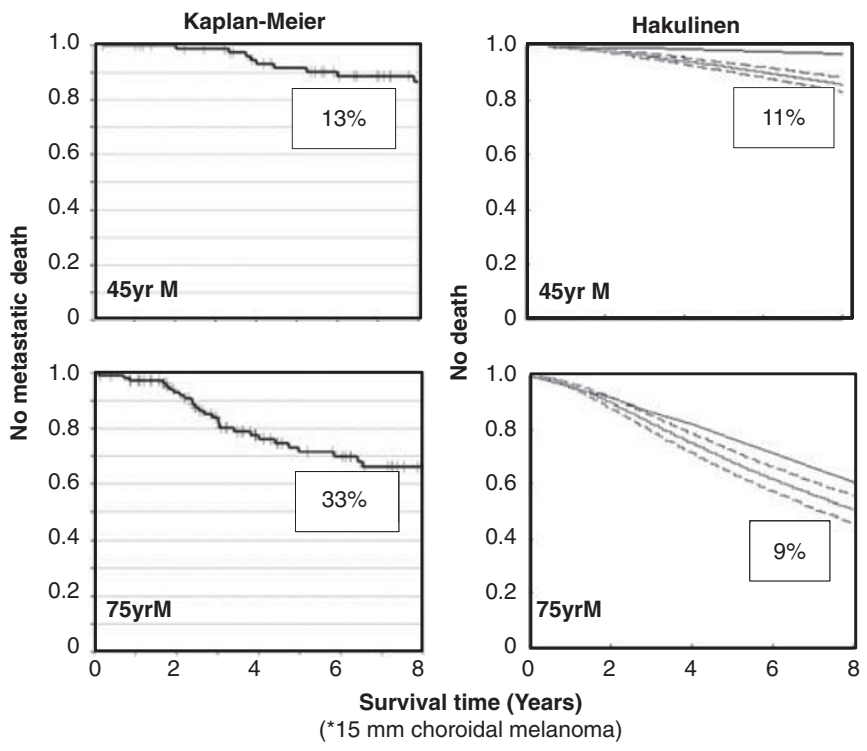

Figure 2 Risk of metastatic death in 45-year-old and 75-year-old men having a 15-mm choroidal melanoma, estimated by the KaplanMeier analysis and using the Hakulinen method. According to the Kaplan-Meier analysis, the risk of metastatic death increases with age from 13 to $33 \%$ whereas with the Hakulinen method these risks diminish from $11 \%$ in young males to $9 \%$ in older men. Adapted from Damato et al. ${ }^{48}$

believes that such arguments are flawed. First of all, in most patients personalised prognostication indicates a better survival probability than patients would otherwise expect, if given only a crude '50-50' estimate; for example, patients with a small, disomy 3 , spindle cell melanoma have a life expectancy that is not significantly worse than the general population. Informing such patients of their good fortune undoubtedly enhances their well being. Second, in-depth psychological studies at the LOOC have shown that patients find an uncertain prognosis more stressful than a poor prognosis, which empowers them to prepare for their illness (eg, organising their personal affairs, deciding about screening, and researching the best centres for treatment of metastastic disease). ${ }^{64}$ Third, personalised prognostication allows special care to be targeted at highrisk patients (ie, counselling, screening, and, in future, systemic adjuvant therapy). Finally, accurate prognostication enhances opportunities for clinical and laboratory research.

\section{Prognostic tumour biopsy}

In 2007, improvements in biopsy techniques, genetic tumour typing, and multivariate analysis encouraged the author to start performing biopsy on patients undergoing radiotherapy or phototherapy. In patients receiving proton beam radiotherapy, the biopsy is performed transretinally on the last day of the radiotherapy or within a week afterwards. This avoids any risk of tumour seeding. In patients having plaque radiotherapy, the biopsy is performed transretinally after completion of the radiotherapy if the tumour is small and posterior, or transsclerally at the time of the plaque insertion if the tumour extends pre-equatorially and if the thickness exceeds $3 \mathrm{~mm}$. The success of tumour biopsy has increased thanks to the improvements in surgical technique and the use of MSA with small samples. As it is not possible to assess mitotic count and extravascular matrix patterns in biopsy specimens, LUMPO was re-developed, with neural networks being replaced by 'accelerated failure time', which copes better with missing data. Prognostic biopsy provides better estimation of the survival proability than reliance on clinical staging alone (Figure 3).

\section{Screening for metastatic disease}

Some authors have suggested that screening for metastatic disease does not confer a survival advantage. ${ }^{65}$ However, new therapies have recently improved survival prospects but are more likely to be effective if undertaken before symptomatic disease develops. Screening also enhances the quality of life of high-risk patients, a normal result reassuring them that they will enjoy good health for many months, at least until the next scan is performed. ${ }^{64}$

Liver function tests tend to become abnormal only when hepatic metastases reach an advanced stage, by which time any opportunities for prolonging life are lost. ${ }^{66}$ 

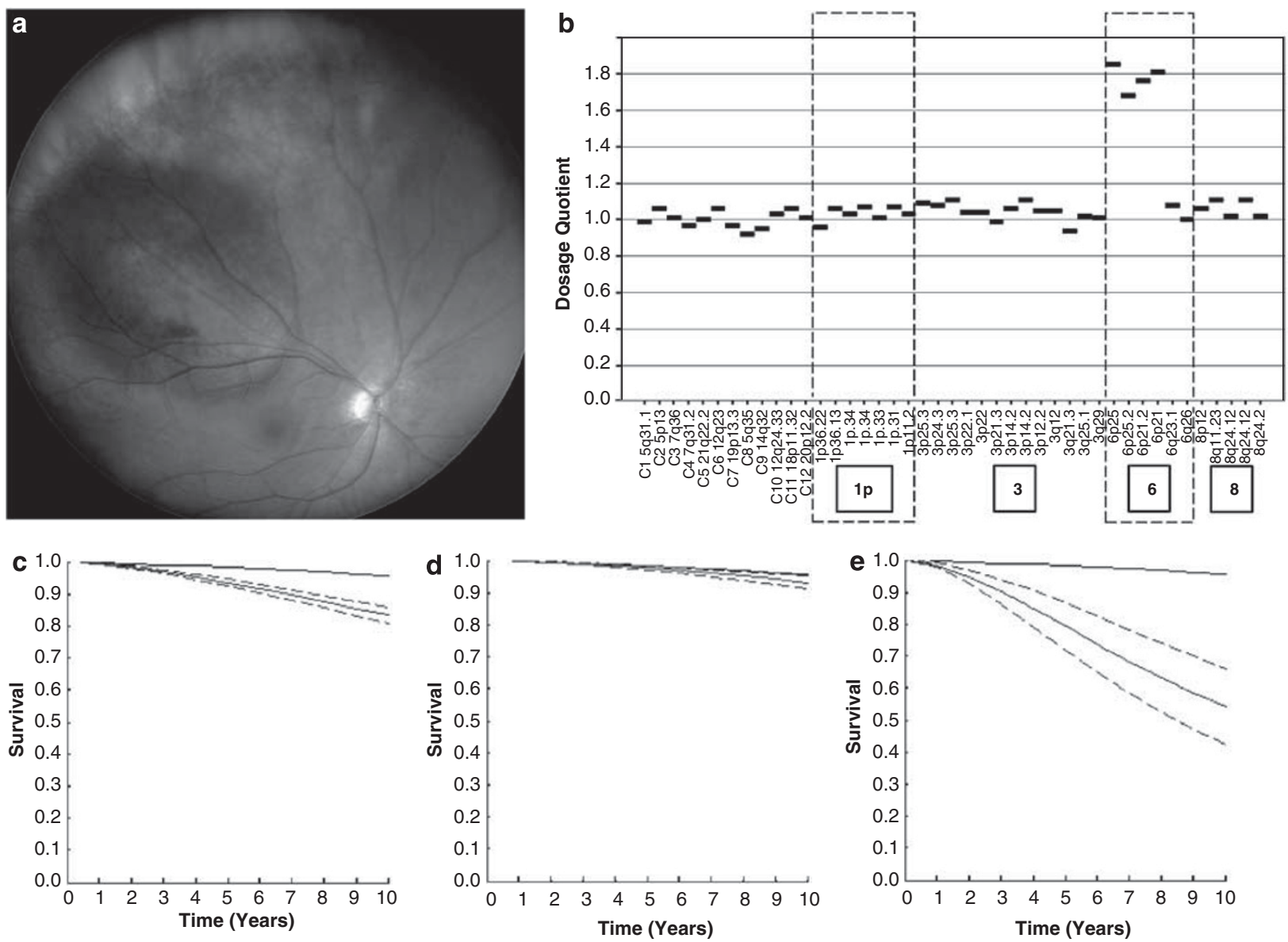

Figure 3 (a) Right eye of a 51-year-old woman with a 14.1-mm diameter choroidal melanoma having a thickness of $2.6 \mathrm{~mm}$. The patient was treated with proton beam radiotherapy and TTT with a good ocular result. (b) MLPA showed the melanoma to be of disomy 3 type, with gains in chromosome $6 \mathrm{p}$ and no gains in 8q. (c) According to LUMPO analysis based on clinical features alone, the 10 -year risk of metastatic death would have been 13\%. (d) Biopsy showed no chromosome 3 loss, indicating this risk of metastatic death to be only 3\%. (e) If the patient had undergone local resection or enucleation and if the tumour had been found to be of monosomy 3 type with epithlioid cells, closed loops and a high-mitotic rate, the 10-year risk of metastatic death would have been as high as $42 \%$.

Liver ultrasonography is the standard method of screening in most centres, because of its relatively low cost; however, its sensitivity is relatively low and depends greatly on the examiner's experience.

Magnetic resonance imaging is currently the most sensitive method. In an audit of high-risk patients receiving ocular treatment of uveal melanoma in Liverpool, hepatic metastases were detected before the onset of symptoms in $92 \%$ of 90 high-risk patients (Marshall et al, unpublished data). Computerised tomography and positron emission tomography (PET) are less sensitive and expose patients to radiation. ${ }^{67,68}$ 18F-FDG PET/CT does not detect metastases from uveal melanoma as sensitively as secondaries from cutaneous melanoma. ${ }^{69}$

Screening with magnetic resonance imaging is relatively expensive and uses up scarce healthcare resources. In a Japanese study, only $9 \%$ of patients were found to have metastases after a mean interval of 5.7 years, a further $4 \%$ having false positive results. ${ }^{70}$ The cost effectiveness of such screening is therefore enhanced by targeting investigations at high-risk patients. Such patient selection would be less effective without personalised prognostication based on multivariate analysis of clinical, histological, and genetic predictors.

\section{Treatment of metastatic disease}

Treatment of metastatic disease from uveal melanoma has been disappointing. ${ }^{71}$ However, a number of new therapies show promise. ${ }^{72}$ Significant prolongation of life has been achieved with intra-hepatic chemotherapy, particularly with isolated liver perfusion with 
melphalan. ${ }^{73}$ Encouraging results have also occurred with partial hepatectomy of isolated metastases, but only a small minority of metastases occur in this manner, most patients developing multiple deposits. ${ }^{74}$ Methods such as radiofrequency and microwave ablation are also effective when only a few metastases are present. Newer agents are currently being investigated and these include ipilimumab and selective internal radiation therapy (SIRT). Ipilumumab is a form of immunotherapy, consisting of a monoclonal antibody that blocks an antigen on cytotoxic T lymphocytes so that these no longer prevent the immune system from killing melanoma cells. ${ }^{75}$ SIRT involves the intrahepatic infusion of radioactive yttrium beads, which accumulate in metastases to provide targeted therapy. ${ }^{76}$ As a result of this progress, more long-term survivors with metastatic disease are being seen than ever before. There is still debate as to whether prolonged survival is the result of treatment or whether it merely reflects earlier detection of metastases (ie, lead-time bias). ${ }^{71}$ Randomised, prospective clinical trials are required to resolve this controversy. In any case, opportunities for evaluating novel treatments have improved considerably thanks to the progress in screening for metastases.

\section{Systemic adjuvant therapy}

It would be ideal if high-risk patients could receive intrahepatic or systemic therapy aimed at suppressing or eradicating micrometastases so as to delay or prevent the onset metastatic disease and death. Such adjuvant therapy has already enhanced survival in patients with breast cancer, orbital rhabdomyosarcoma, and a growing number of other cancers. Whitehead et al have analysed the Liverpool data to estimate the number of patients that would need to be enrolled to have a $90 \%$ power to detect a $10 \%$ increase in survival from $50 \%$ to $60 \%$ in high-risk patients with monosomy 3 melanoma exceeding $15 \mathrm{~mm}$ in basal tumour diameter (Whitehead et al, unpublished data). A standard randomised trial would require recruitment of almost a thousand patients and a trial duration of $>6$ years.

It may be possible to evaluate systemic adjuvant therapy in high-risk patients with uveal melanoma by measuring the effect of treatment on circulating tumour cells, as has already been achieved with other cancers. The accuracy of such measurements has recently improved with the development of semi-automated systems such as Veridex (Veridex, Raritan, NJ, USA). In collaboration with the Paterson Institute at Manchester, Angi et al have successfully detected circulating tumour cells in patients with uveal melanoma treated in Liverpool (Angi et al, unpublished data).

\section{Conclusions}

The management of patients with uveal melanoma has advanced significantly as a result of progress in pathology, ocular investigation, ocular treatment, prognostication, and treatment of metastatic disease. Patient care has also improved because of enhanced counselling and psychological support. A key to progress has been the formation of multidisciplinary teams working in specialised, supraregional ocular oncology centres. There is scope for improvement in the early detection, diagnosis, and referral of patients with uveal melanoma.

\section{Conflict of interest}

The author declares no conflict of interest.

\section{Acknowledgements}

We acknowledge Marcela Baudo (Scientist); Jenny Burke (Secretary); Gary Cheetham (Data Manager); Sarah Coupland (Pathologist); Antonio Eleuteri (Statistician); R Doug Errington (Radiotherapist); Jonathan Evans (Interventional radiologist); Carl Groenewald (Vitreoretinal surgeon); Gwen Hachuela (Nurse); Gill Hebbar (Nurse); Heinrich Heimann (Ocular Oncologist); Laura Hope-Stone (Health Psychologist); Andy Jones (Anaesthetist); Andrzej Kacperek (Radiation Physicist); Helen Kalirai (Scientist); Sam Kaye (Nurse); Paula Maxhuni (Clerk); Helen and Peter Mayles (Radiation Physicists); Nicola Longrigg (Compliance Officer); Dawn Roberts (Research Secretary); Julie Sudlow (Personal Assistant); and Azzam Taktak (Mathematician). We also acknowledge the National Specialist Commissioning Group, which funded the Liverpool Ocular Oncology Service.

\section{References}

1 Damato BE, Coupland SE. Differences in uveal melanomas between men and women from the British Isles. Eye 2012; 26: 292-299.

2 Damato EM, Damato BE. Detection and time to treatment of uveal melanoma in the United Kingdom: an evaluation of 2384 patients. Ophthalmology 2012; e-pub ahead of print 11 April 2012; doi:10.1016/j.ophtha.2012.01.048.

3 Damato BE, Foulds WS. Tumour-associated retinal pigment epitheliopathy. Eye 1990; 4: 382-387.

4 Coupland SE, Campbell I, Damato B. Routes of extraocular extension of uveal melanoma: risk factors and influence on survival probability. Ophthalmology 2008; 115: 1778-1785.

5 Eskelin S, Pyrhönen S, Hahka-Kemppinen M, Tuomaala S, Kivelä T. A prognostic model and staging for metastatic uveal melanoma. Cancer 2003; 97: 465-475. 
6 Anderson MF, Callejo S, Bridson JD, Damato BE. Diagnostic information provided by referrers to patients with suspected uveal melanoma. Eye 2011; 25: 392.

7 Bridson JD, Damato B. Consent to rapid treatment of eye tumours: is the waiting time too short at Liverpool Ocular Oncology Centre? Clin Ethics 2010; 5(2): 86-94.

8 Feinstein EG, Marr BP, Winston CB, Abramson DH. Hepatic abnormalities identified on abdominal computed tomography at diagnosis of uveal melanoma. Arch Ophthalmol 2010; 128: 319-323.

9 Ah-Fat FG, Sharma MC, Damato BE. Taping outpatient consultations: a survey of attitudes and responses of adult patients with ocular malignancy. Eye 1998; 12: 789-791.

10 Damato B. Does ocular treatment of uveal melanoma influence survival? Br J Cancer 2010; 103: 285-290.

11 Knapp H. A treatise on intraocular tumors, from original clinical observations and anatomical investigations. English translation; (edn) William Wood \& Co.: New York, 1869.

12 Manschot WA, van Strik R. Is irradiation a justifiable treatment of choroidal melanoma? Analysis of published results. Br J Ophthalmol 1987; 71: 348-352.

13 Zimmerman LE, McLean IW, Foster WD. Does enucleation of the eye containing a malignant melanoma prevent or accelerate the dissemination of tumour cells. Br JOphthalmol 1978; 62: 420-425.

14 The COMS randomized trial of iodine 125 brachytherapy for choroidal melanoma: V. Twelve-year mortality rates and prognostic factors: COMS report no. 28. Arch Ophthalmol 2006; 124: 1684-1693.

15 Hawkins BS. The Collaborative Ocular Melanoma Study (COMS) randomized trial of pre-enucleation radiation of large choroidal melanoma: IV. Ten-year mortality findings and prognostic factors. COMS report number 24. Am J Ophthalmol 2004; 138: 936-951.

16 Damato B. Legacy of the collaborative ocular melanoma study. Arch Ophthalmol 2007; 125: 966-968.

17 Eskelin S, Pyrhönen S, Summanen P, Hahka-Kemppinen M, Kivelä T. Tumor doubling times in metastatic malignant melanoma of the uvea: tumor progression before and after treatment. Ophthalmology 2000; 107: 1443-1449.

18 Prescher G, Bornfeld N, Hirche H, Horsthemke B, Jöckel $\mathrm{KH}$, Becher R. Prognostic implications of monosomy 3 in uveal melanoma. Lancet 1996; 347: 1222-1225.

19 Höglund M, Gisselsson D, Hansen GB, White VA, Säll T, Mitelman F et al. Dissecting karyotypic patterns in malignant melanomas: temporal clustering of losses and gains in melanoma karyotypic evolution. Int J Cancer 2004; 108: $57-65$

20 Tschentscher F, Husing J, Holter T, Kruse E, Dresen IG, Jöckel KH et al. Tumor classification based on gene expression profiling shows that uveal melanomas with and without monosomy 3 represent two distinct entities. Cancer Res 2003; 63: 2578-2584.

21 Damato B, Dopierala JA, Coupland SE. Genotypic profiling of 452 choroidal melanomas with multiplex ligationdependent probe amplification. Clin Cancer Res 2010; 16: 6083-6092.

22 Callejo SA, Dopierala J, Coupland SE, Damato B. Sudden growth of a choroidal melanoma and multiplex ligationdependent probe amplification findings suggesting late transformation to monosomy 3 type. Arch Ophthalmol 2011; 129: 958-960.
23 Meyer Schwickerath G, Vogel M. Treatment of malignant melanomas of the choroid by photocoagulation. Trans Ophthalmol Soc UK 1977; 97: 416-420.

24 Foulds WS, Damato BE. Low-energy long-exposure laser therapy in the management of choroidal melanoma. Graefes Arch Clin Exp Ophthalmol 1986; 224: 26-31.

25 Oosterhuis JA, Journee-de Korver HG, Kakebeeke-Kemme HM, Bleeker JC. Transpupillary thermotherapy in choroidal melanomas. Arch Ophthalmol 1995; 113: 315-321.

26 Lang GE, Mennel S, Spital G, Wachtlin J, Jurklies B, Heimann $\mathrm{H}$ et al. Different indications of photodynamic therapy in ophthalmology. Klin Monatsbl Augenheilkd 2009; 226: 725-739.

27 Moore RF. Choroidal sarcoma treated by the intraocular insertion of radon seeds. Br J Ophthalmol 1930; 14: 145-152.

28 Russo A, Laguardia M, Damato B. Eccentric ruthenium plaque radiotherapy of posterior choroidal melanoma. Graefes Arch Clin Exp Ophthalmol 2012; e-pub ahead of print 28 March 2012; PMID 22460679.

29 Damato B, Patel I, Campbell IR, Mayles HM, Errington RD. Visual acuity after Ruthenium(106) brachytherapy of choroidal melanomas. Int J Radiat Oncol Biol Phys 2005; 63: 392-400.

30 Damato B, Patel I, Campbell IR, Mayles HM, Errington RD. Local tumor control after (106)Ru brachytherapy of choroidal melanoma. Int J Radiat Oncol Biol Phys 2005; 63: 385-391.

31 Damato B, Kacperek A, Chopra M, Campbell IR, Errington RD. Proton beam radiotherapy of choroidal melanoma: the Liverpool-Clatterbridge experience. Int J Radiat Oncol Biol Phys 2005; 62: 1405-1411.

32 Daftari IK, Char DH, Verhey LJ, Castro JR, Petti PL, Meecham WJ. Anterior segment sparing to reduce charged particle radiotherapy complications in uveal melanoma. Int J Radiat Oncol Biol Phys 1997; 39: 997-1010.

33 Damato B, Kacperek A, Chopra M, Sheen MA, Campbell IR, Errington RD. Proton beam radiotherapy of iris melanoma. Int J Radiat Oncol Biol Phys 2005; 63: 109-115.

34 Tan AN, Hoevenaars JG, Webers CA, Damato B, Beckers HJ. Baerveldt implant for secondary glaucoma due to iris melanoma. Clin Ophthalmol 2010; 4: 407-409.

35 Damato EM, Damato B, Sibbring JS, Coupland SE. Ciliary body melanoma with partial deletion of chromosome 3 detected with multiplex ligation-dependent probe amplification. Graefes Arch Clin Exp Ophthalmol 2008; 246: 1637-1640.

36 Foulds WS. The local excision of choroidal melanomata. Trans Ophthalmol Soc UK 1973; 93: 343-346.

37 Damato BE, Paul J, Foulds WS. Risk factors for residual and recurrent uveal melanoma after trans-scleral local resection. Br J Ophthalmol 1996; 80: 102-108.

38 Damato BE, Paul J, Foulds WS. Risk factors for metastatic uveal melanoma after trans-scleral local resection. $\mathrm{Br} J$ Ophthalmol 1996; 80: 109-116.

39 Damato BE, Paul J, Foulds WS. Predictive factors of visual outcome after local resection of choroidal melanoma. $\mathrm{Br} \mathrm{J}$ Ophthalmol 1993; 77: 616-623.

40 Damato B, Groenewald CP, McGalliard JN, Wong D. Rhegmatogenous retinal detachment after transscleral local resection of choroidal melanoma. Ophthalmology 2002; 109: 2137-2143.

41 Damato B, Groenewald C, McGalliard J, Wong D. Endoresection of choroidal melanoma. Br J Ophthalmol 1998; 82: 213-218. 
42 Hadden PW, Hiscott PS, Damato BE. Histopathology of eyes enucleated after endoresection of choroidal melanoma. Ophthalmology 2004; 111: 154-160.

43 Damato B, Wong D, Green FD, Mackenzie JM. Intrascleral recurrence of uveal melanoma after transretinal "endoresection". Br J Ophthalmol 2001; 85: 114-115.

44 Bechrakis NE, Foerster MH. Neoadjuvant proton beam radiotherapy combined with subsequent endoresection of choroidal melanomas. Int Ophthalmol Clin 2006; 46: 95-107.

45 Schilling H, Bornfeld N, Talies S, Anastassiou G, Schüler A, Horstmann GA. [Endoresection of large uveal melanomas after pretreatment by single-dose stereotactic convergence irradiation with the leksell gamma knife-first experience on 46 cases]. Klin Monbl Augenheilkd 2006; 223: 513-520.

46 Damato B, Lecuona K. Conservation of eyes with choroidal melanoma by a multimodality approach to treatment: an audit of 1632 patients. Ophthalmology 2004; 111: 977-983.

47 Sandinha T, Hebbar G, Kenawy N, Hope-Stone L, Damato B. A nurse-led ocular oncology clinic in Liverpool: results of a 6-month trial. Eye 2012; e-pub ahead of print 20 April 2012; doi:10.1038/eye.2012.62.

48 Damato B, Eleuteri A, Taktak AF, Coupland SE. Estimating prognosis for survival after treatment of choroidal melanoma. Prog Retin Eye Res 2011; 30: 285-295.

49 Damato B, Coupland SE. A reappraisal of the significance of largest basal diameter of posterior uveal melanoma. Eye 2009; 23: 2152-2160.

50 Shields CL, Furuta M, Thangappan A, Nagori S, Mashayekhi A, Lally DR et al. Metastasis of uveal melanoma millimeter-by-millimeter in 8033 consecutive eyes. Arch Ophthalmol 2009; 127: 989-998.

51 Finger PT. The 7th edition AJCC staging system for eye cancer: an international language for ophthalmic oncology. Arch Pathol Lab Med 2009; 133: 1197-1198.

52 Jmor F, Kalirai H, Taktak A, Damato B, Coupland SE. HSP27 protein expression in uveal melanoma: correlation with predicted survival. Acta Ophthalmol 2010; 10-3768.

53 Harbour JW, Onken MD, Roberson ED, Duan S, Cao L, Worley LA et al. Frequent mutation of BAP1 in metastasizing uveal melanomas. Science 2010; 330: 1410-1413.

54 Angi M, Damato B, Kalirai H, Dodson A, Taktak A, Coupland SE. Immunohistochemical assessment of mitotic count in uveal melanoma. Acta Ophthalmol 2011; 89: e155-e160.

55 Damato B, Duke C, Coupland SE, Hiscott P, Smith PA, Campbell I et al. Cytogenetics of uveal melanoma: a 7-year clinical experience. Ophthalmology 2007; 114: 1925-1931.

56 Lake SL, Kalirai H, Dopierala J, Damato BE, Coupland SE. Comparison of formalin-fixed and snap-frozen samples analyzed by multiplex ligation-dependent probe amplification for prognostic testing in uveal melanoma. Invest Ophthalmol Vis Sci 2012; 53: 2647-2652.

57 Damato B, Dopierala J, Klaasen A, van Dijk M, Sibbring J, Coupland SE. Multiplex ligation-dependent probe amplification of uveal melanoma: correlation with metastatic death. Invest Ophthalmol Vis Sci 2009; 50: 3048-3055.

58 Dopierala J, Damato BE, Lake SL, Taktak AF, Coupland SE. Genetic heterogeneity in uveal melanoma assessed by multiplex ligation-dependent probe amplification. Invest Ophthalmol Vis Sci 2010; 51: 4898-4905.
59 Scholes AG, Damato BE, Nunn J, Hiscott P, Grierson I, Field JK. Monosomy 3 in uveal melanoma: correlation with clinical and histologic predictors of survival. Invest Ophthalmol Vis Sci 2003; 44: 1008-1011.

60 Onken MD, Worley LA, Ehlers JP, Harbour JW. Gene expression profiling in uveal melanoma reveals two molecular classes and predicts metastatic death. Cancer Res 2004; 64: 7205-7209.

61 Onken MD, Worley LA, Tuscan MD, Harbour JW. An accurate, clinically feasible multi-gene expression assay for predicting metastasis in uveal melanoma. J Mol Diagn 2010; 12: 461-468.

62 Eleuteri A, Damato B, Coupland SE, Taktak AF. Enhancing survival prognostication in patients with choroidal melanoma by integrating pathologic, clinical and genetic predictors of metastasis. Int J Biomed EngTechnol 2012; 8: 18-35.

63 Hakulinen T, Teppo L, Saxen E. Cancer of the eye, a review of trends and differentials. World Health Stat Q 1978; 31: 143-158.

64 Cook SA, Damato B, Marshall E, Salmon P. Psychological aspects of cytogenetic testing of uveal melanoma: preliminary findings and directions for future research. Eye 2009; 23: 581-585.

65 Kim IK, Lane AM, Gragoudas ES. Survival in patients with presymptomatic diagnosis of metastatic uveal melanoma. Arch Ophthalmol 2010; 128: 871-875.

66 Diener-West M, Reynolds SM, Agugliaro DJ, Caldwell R, Cumming K, Earle JD. Screening for metastasis from choroidal melanoma: The Collaborative Ocular Melanoma Study Group Report 23. J Clin Oncol 2004; 22: 2438-2444.

67 Servois V, Mariani P, Malhaire C, Petras S, PipernoNeumann S, Plancher C et al. Preoperative staging of liver metastases from uveal melanoma by magnetic resonance imaging (MRI) and fluorodeoxyglucose-positron emission tomography (FDG-PET). Eur J Surg Oncol 2010; 36: 189-194.

68 Orcurto V, Denys A, Voelter V et al. (18)F-fluorodeoxyglucose positron emission tomography/computed tomography and magnetic resonance imaging in patients with liver metastases from uveal melanoma: results from a pilot study. Melanoma Res 2012; 22: 63-69.

69 Strobel K, Bode B, Dummer R, Schalenbourg A, Schnyder P, Zografos L. Limited value of 18F-FDG PET/CT and S-100B tumour marker in the detection of liver metastases from uveal melanoma compared to liver metastases from cutaneous melanoma. Eur J Nucl Med Mol Imaging 2009; 36: 1774-1782.

70 Maeda T, Tateishi U, Suzuki S, Arai Y, Kim EE, Sugimura K. Magnetic resonance screening trial for hepatic metastasis in patients with locally controlled choroidal melanoma. Jpn J Clin Oncol 2007; 37: 282-286.

71 Augsburger JJ, Correa ZM, Shaikh AH. Effectiveness of treatments for metastatic uveal melanoma. Am J Ophthalmol 2009; 148: 119-127.

72 Sato T. Locoregional management of hepatic metastasis from primary uveal melanoma. Semin Oncol 2010; 37: 127-138.

73 Alexander HR Jr., Libutti SK, Pingpank JF, Steinberg SM, Bartlett DL, Helsabeck C. Hyperthermic isolated hepatic perfusion using melphalan for patients with ocular melanoma metastatic to liver. Clin Cancer Res 2003; 9: 6343-6349. 
74 Mariani P, Piperno-Neumann S, Servois V, Berry MG, Dorval T. Surgical management of liver metastases from uveal melanoma: 16 years' experience at the Institut Curie. Eur J Surg Oncol 2009; 35: 1192-1197.

75 Hodi FS, O'Day SJ, McDermott DF, Weber RW, Sosman JA, Haanen JB et al. Improved survival with ipilimumab in patients with metastatic melanoma. N Engl J Med 2010; 363:

711-723.

76 Klingenstein A, Haug AR, Zech CJ, Schaller UC. Radioembolization as locoregional therapy of hepatic metastases in uveal melanoma patients. Cardiovasc Intervent Radiol 2012; e-pub ahead of print 21 April 2011; PMID: 22526099. 